\title{
THE INCIDENCE CHROMATIC NUMBER OF SOME GRAPH
}

\author{
LIU XIKUI AND LI YAN
}

Received 1 April 2003 and in revised form 5 December 2003

The concept of the incidence chromatic number of a graph was introduced by Brualdi and Massey (1993). They conjectured that every graph $G$ can be incidence colored with $\Delta(G)+2$ colors. In this paper, we calculate the incidence chromatic numbers of the complete $k$-partite graphs and give the incidence chromatic number of three infinite families of graphs.

\section{Introduction}

Throughout the paper, all graphs dealt with are finite, simple, undirected, and loopless. Let $G$ be a graph, and let $V(G), E(G), \Delta(G)$, respectively, denote vertex set, edge set, and maximum degree of G. In 1993, Brualdi and Massey [3] introduced the concept of incidence coloring. The order of $G$ is the cardinality $|v(G)|$. The size of $G$ is the cardinality $|E(G)|$. Let

$$
I(G)=\{(v, e) \mid v \in V, e \in E, v \text { is incident with } e\}
$$

be the set of incidences of $G$. We say that two incidences $(v, e)$ and $(w, f)$ are adjacent provided one of the following holds:

(i) $v=w$;

(ii) $e=f$;

(iii) the edge $v w=e$ or $v w=f$.

Figure 1.1 shows three cases of two incidences being adjacent.

An incidence coloring $\sigma$ of $G$ is a mapping from $I(G)$ to a set $C$ such that no two adjacent incidences of $G$ have the same image. If $\sigma: I(G) \rightarrow C$ is an incidence coloring of $G$ and $|C|=k, k$ is a positive integer, then we say that $G$ is $k$-incidence colorable. 


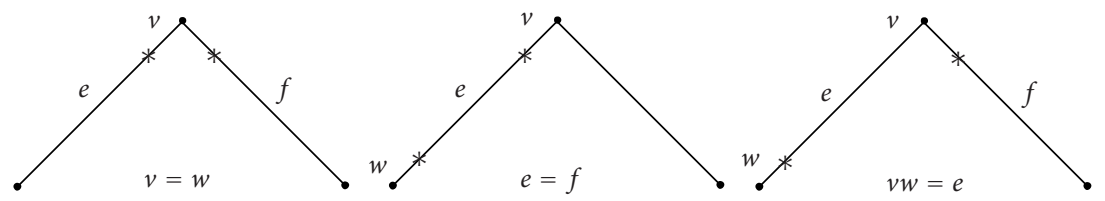

(a)

(b)

(c)

Figure 1.1. Cases of two incidences being adjacent.

The minimum cardinality of $C$ for which there exists an incidence coloring $\sigma: I(G) \rightarrow C$ is called the incidence chromatic number of $G$, and is denoted by $\operatorname{inc}(G)$. A partition $\left\{I_{1}, I_{2}, \ldots, I_{k}\right\}$ of $I(G)$ is called an independence partition of $I(G)$ if each $I_{i}$ is independent in $I(G)$ (i.e., no two incidences of $I_{i}$ are adjacent in $I(G)$ ). Clearly, for $k^{\prime} \geq \operatorname{inc}(G), G$ is $k^{\prime}$-incidence colorable.

We may consider $G$ as a digraph by splitting each edge $u v$ into two opposite arcs $(u, v)$ and $(v, u)$. Let $e=u v$. We identify $(u, e)$ with the $\operatorname{arc}(u, v)$. So $I(G)$ may be identified with the set of all $\operatorname{arcs} A(G)$. Two distinct arcs (incidences) $(u, v)$ and $(x, y)$ are adjacent if one of the following holds (see Figure 1.2):

(1') $u=x$;

(2') $u=y$ and $v=x$;

(3') $v=x$.

This concept was first developed by Brualdi and Massey [3] in 1993. They posed the incidence coloring conjecture (ICC), which states that for every graph $G$, inc $(G) \leq$ $\Delta+2$. In 1997, Guiduli [5] showed that incidence coloring is a special case of directed star arboricity, introduced by Algor and Alon [1]. They pointed out that the ICC was solved in the negative following an example in [1]. Following the analysis in [1], they showed that $\operatorname{inc}(G) \geq \Delta+\Omega(\log \Delta)$, where $\Omega=1 / 8-\circ(1)$. Making use of a tight upper bound for directed star arboricity, they obtained the upper bound $\operatorname{inc}(G) \leq \Delta+$ $O(\log \Delta)$.

Brualdi and Massey determined the incidence chromatic numbers of trees, complete graphs, and complete bipartite graphs [3]; Chen, Liu, and Wang determined the incidence chromatic numbers of paths, cycles, fans, wheels, adding-edge wheels, and complete 3partite graphs [4].

In this paper, we will consider the incidence chromatic number for complete $k$-partite graphs. We will give the incidence chromatic number of complete $k$-partite graphs, and also give the incidence chromatic number of three infinite families of graphs. Let $k$ be positive integer, put $[k]=1,2, \ldots, k$. We state first the following definitions.

Definition 1.1. For a graph $G(V, E)$ with vertex set $V$ and edge set $E$, the incidence graph $I(G)$ of $G$ is defined as the graph with vertex set $V(I(G))$ and edge set $E(I(G))$.

Definitions not given here may be found in [2]. 


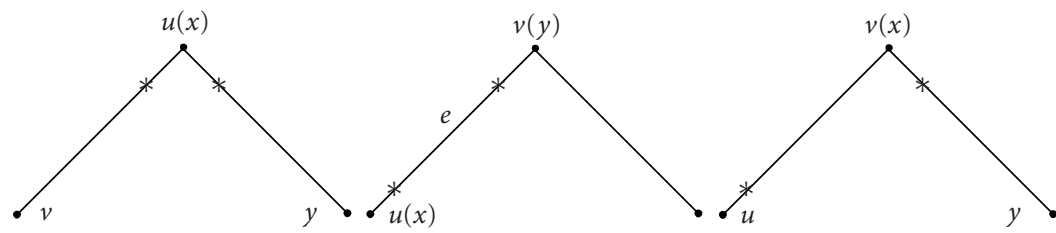

(a)

(b)

(c)

Figure 1.2. Cases of two arcs (incidences) are adjacent.

\section{Some useful lemmas and properties of incidence chromatic number}

Lemma 2.1. Let $T$ be a tree of order $n \geq 2$ with maximum degree $\Delta$. Then $\operatorname{inc}(T)=\Delta+1$.

Lemma 2.2. A graph $G$ is $k$-incidence colorable if and only if its incidence graph $I(G)$ is $k$-vertex colorable, that is, inc $(G)=\chi(I(G))$.

Let $M=\{(u e, v e) \mid e=u v \in E(G),(u e, v e) \in E(I(G))\}$, then $M$ forms a perfect matching of incidence graph $I(G)$. The following lemmas are obvious.

Lemma 2.3. The incidence graph $I(G)$ of a graph $G$ is a graph with a perfect matching.

Lemma 2.4. For a graph $G, v \in V(G)$, let $B_{v}=\{(u, u v) \mid u v \in E(G), u \in V(G)\}, A_{v}=$ $\{(v, v u) \mid u v \in E(G), u \in V(G)\}$, then $\left\{B_{v}\right\}$ is an independence-partition of incidence graph $I(G)$, and the induced subgraph $G\left[A_{v}\right]$ of $I(G)$ is a clique graph.

By the definition of incidence graph, it is easy to give the proof.

Lemma 2.5. Let $\Delta$ be the maximum degree of graph $G, I(G)$ the incidence graph, then complete graph $K_{\Delta+1}$ is a subgraph of $I(G)$.

Proof. Let $d(u)=\Delta, p=\Delta$, and $e_{k}=u v_{1}, e_{2}=u v_{2}, \ldots, e_{p}=u v_{1}$ be the edges of $G . p$ incidences in $I_{u}=\left\{\left(u, e_{1}\right),\left(u, e_{2}\right), \ldots,\left(u, e_{p}\right)\right\}$ are adjacent to each other. For an incidence in $I_{v}=\left\{\left(v_{i}, v_{i} u\right) \mid u v_{i} \in G, 1 \leq i \leq p\right\},\left(v_{i}, v_{i} u\right)$ is adjacent to all incidences in $I_{u}$. Since $p+$ 1 incidences $\left(u, e_{2}\right), \ldots,\left(u, e_{p}\right),\left(v_{i}, v_{i} u\right)$ are vertices of $I(G)$, by the definition of incidence graph, we can complete the proof.

Lemma 2.6. For a simple graph $G$ with order $n$, inc $(G)=n=\Delta(G)+1$, when $\Delta(G)=n-1$.

Proof. Let $|V(G)|=\Delta+1=v(G)$, by Lemma 2.4, $\left\{B_{v}\right\}$ is an independence-partition of incidence graph $I(G)$, then $\chi(I(G)) \leq v(G)$. By Lemma 2.5, $K_{\Delta+1}$ is the subgraph of $I(G)$, thus $\chi(I(G)) \geq v(G)$, then $\operatorname{inc}(G)=\chi(I(G))=\Delta+1$, as required.

The following corollaries can be easily verified.

Corollary 2.7. Let $G$ be a graph with order $n(n \geq 2)$, then $\operatorname{inc}(G) \leq \Delta+2$, when $\Delta(G)=$ $n-2$. 
In fact, for graphs $G$ with order $n$, we can give each incidence in $I(G)$ proper incidence coloring as follows. Let $V(G)=\left\{v_{1}, v_{2}, \ldots, v_{n}\right\}$ be the vertex set and $C=\{1,2, \ldots, n\}$ the color set. For $i, j=1,2, \ldots, n$, we let $\sigma\left(v_{i}, v_{i} v_{j}\right)=j$. It is easy to see that the coloring above is an incidence coloring of $G$ only with $n$ colors. That is, $\operatorname{inc}(G) \leq \Delta+2$, when $\Delta \geq n-2$.

Corollary 2.8. Let $W_{n}$ be the wheel graph with order $n+1$. Then $\operatorname{inc}\left(W_{n}\right)=n+1$.

Lemma 2.9. Let $H$ be a subgraph of $G$, then $\operatorname{inc}(H) \leq G$.

Lemma 2.10. Let $G$ be union of disjoint graphs $G_{1}, G_{2}, \ldots$, and $G_{t}$. If $G_{i}$ has an $m$-incidence coloring for all $i=1,2, \ldots, t$, then $G$ has an $m$-incidence coloring. That is $\operatorname{inc}(G)=$ $\max \left\{\operatorname{inc}\left(G_{i}\right) \mid i=1,2, \ldots, t\right\}$.

Proof. To prove this lemma, we only need to prove that $G_{1} \cup G_{2}$ has an $m$-incidence coloring. Let $\left\{I_{1}, I_{2}, \ldots, I_{m}\right\}$ be an independence partition of $I\left(G_{1}\right)$, and $\left\{I_{1}^{\prime}, I_{2}^{\prime}, \ldots, I_{m}^{\prime}\right\}$ an independence partition of $I\left(G_{2}\right)$. Then $\left\{I_{1} \cup I_{1}^{\prime}, I_{2} \cup I_{2}^{\prime}, \ldots, I_{m} \cup I_{m}^{\prime}\right\}$ forms an independence-partition of $I\left(G_{1}\right) \cup I\left(G_{2}\right)$. Hence $G$ has an $m$-incidence coloring. The proof of the lemma is complete.

Theorem 2.11. Let $G$ be a graph with maximum degree $\Delta(G)=n-2$ and minimum degree $\delta(G) \leq[n / 2]-1$, then $\operatorname{inc}(G)=n-1=\Delta(G)+1$.

Proof. Let $V(G)=\left\{v_{1}, v_{2}, \ldots, v_{n}\right\}, d\left(v_{1}\right)=\delta(G)$, and $u \notin V(G)$. Consider the auxiliary graph $G^{\prime}$ with vertex set $V\left(G^{\prime}\right)=V(G) \cup\{u\}$ and edge set $E\left(G^{\prime}\right)=E(G) \cup\left\{u v_{i} \mid i=\right.$ $2,3, \ldots, n\}$. It follows that $\Delta\left(G^{\prime}\right)=n-1$. Let $G^{\prime \prime}=G^{\prime}-\left\{v_{1}\right\}$, then $\Delta\left(G^{\prime \prime}\right)=n-1$, by Lemma 2.5, $\operatorname{inc}\left(G^{\prime \prime}\right)=n$. For color set $C=\{1,2, \ldots, n\}$, suppose that $\sigma^{\prime}$ is the $n$-incidence coloring of $G^{\prime \prime}$ with color set $C$. Without loss of generality, let $\sigma^{\prime}\left(v_{i}, v_{i} v_{j}\right)=j\left(v_{i} v_{j} \in\right.$ $E(G))$ and $\sigma^{\prime}\left(v_{i}, v_{i} u\right)=1(i=2,3, \ldots, n), \sigma^{\prime}\left(u, u v_{i}\right)=i(i=2,3, \ldots, n)$. In incidence set $I(G)$, incidences $\left(v_{i}, v_{i} v_{j}\right)(i, j=2,3, \ldots, n$, and $i \neq j)$ are all adjacent to $\left(v_{i}, v_{i} u\right)$ and $\left(v_{j}, v_{j} u\right)$, thus the color $n$ cannot be used to color any incidence in $I\left(G^{\prime \prime}-\{u\}\right)$. Denote by $N\left(v_{1}\right)=\left\{v_{i_{1}}, v_{i_{2}}, \ldots, v_{i_{\delta}}\right\}$ the vertices adjacent to $v_{1}$. The incidence coloring $\sigma^{\prime}$ of graph $G^{\prime \prime}$ may be extended to an incidence coloring $\sigma$ of graph $G$. For $x, y \in V(G)$ and $x, y \notin\left\{v_{1}\right\} \cup N\left(v_{1}\right)$, let $\sigma(x, x y)=\sigma^{\prime}(x, x y)$. Because $\Delta(G)=n-2$, for vertex $v_{i_{k}}(k=$ $1,2, \ldots, \delta)$, there exists a vertex $v_{t_{k}} \in V(G)$ such that $v_{i_{k}} v_{t_{k}} \notin E(G)$. Let $\sigma\left(v_{i_{k}}, v_{i_{k}} v_{1}\right)=t_{k}$. At last, we give incidences $\left(v_{1}, v_{1}, v_{i_{k}}\right) \in I(G)(k=1,2, \ldots, \delta)$ the color used to color incidence $\left(u, u v_{i}\right) \in I\left(G^{\prime \prime}\right)(i=2,3, \ldots, n)$. Since $d\left(v_{1}\right)=\delta \leq[n / 2]-1$, then $2 d\left(v_{1}\right) \leq 2[n / 2]-2 \leq$ $n-2$, that is, $d\left(v_{1}\right) \leq n-2-d\left(v_{1}\right)$, thus we can select $d\left(v_{1}\right)$ colors to incidence color, thus $\sigma$ is a proper $n$-incidence coloring of $G$. The proof is completed.

For the general case, using the way similar to Theorem 2.11, we can give a stronger result.

Theorem 2.12. For graph $G$ with order $n$ and maximum degree $\Delta(G)=n-k$, inc $(G)$ $=n-k+1=\Delta(G)+1$, when minimum degree $\delta(G) \leq[(n-k+2) / 2]-1$.

For a graph $G$, if there exists two vertices $u, v \in V(G) \backslash v_{1}$ such that $d(u)=n-3, d(v) \leq$ $n-4$, and $u v \notin E(G)$, we say that $G$ is with the property $P$. 
Theorem 2.13. For graph $G$ with order $n$ and maximum degree $\Delta(G)=n-3$, inc $(G)$ $\leq \Delta(G)+2(n \geq 4)$, when minimum degree $\delta(G) \leq[n / 2]-1$.

Proof. By $V_{n}=\left\{v_{1}, v_{2}, \ldots, v_{n}\right\}$ we denote a labeling of the vertices of $G$ and let $d\left(v_{1}\right)=$ $\delta(G)$. For $n=4,5$, the desired result follows from Lemma 2.1.

For the case $n \geq 6$, the proof can be divided into two cases.

Case 1. $G$ is with the property $P$. Consider the auxiliary graph $G^{\prime}=G+u v$. Since $\Delta\left(G^{\prime}\right)=$ $n-2$ and $\delta\left(G^{\prime}\right) \leq[n / 2]-1$, by Theorem 2.11, inc $\left(G^{\prime}\right)=n-1=\Delta\left(G^{\prime}\right)+1$. Thus inc $(G) \leq$ $\operatorname{inc}\left(G^{\prime}\right)=\Delta(G)+2$.

Case 2. $G$ not with the property $P$. For two vertices $u, v \in V(G) \backslash v_{1}$, let $V_{1}(G)=\{v \in$ $\left.V(G) \mid d_{G}(v)=n-3\right\}$ and $V_{2}(G)=V(G) \backslash\left\{V_{1}(G) \cup\left\{v_{1}\right\}\right\}$.

Subcase 1. $V_{2}(G)=\varnothing$. Let $w \notin V(G)$ and $G^{\prime}=G+w+\left\{w v \mid v \in V_{1}(G)\right\}$, then $\Delta\left(G^{\prime}\right)=$ $n-1$ and $\delta\left(G^{\prime}\right) \leq[n / 2]-1$. By Theorem 2.11, using similar methods as in the proof of Theorem 2.11, we can prove the desired $\operatorname{result} \operatorname{inc}(G) \leq n-1$.

Subcase 2. $V_{2}(G) \neq \varnothing$. Let $x$ be the arbitrary vertex in $V_{1}(G)$, then $N(x)=V_{2}(G)$. For arbitrary vertex $v \in V_{2}(G)$, since $d(v) \leq n-4$, then $\left|V_{2}(G)\right| \geq 3$, and there exists two vertices $u_{1}^{\prime}, v_{1}^{\prime}$ in $V_{2}(G)$ such that $u_{1}^{\prime} v_{1}^{\prime} \notin E(G)$. Let $G_{1}=G+u_{1}^{\prime} v_{1}^{\prime}$. If $G_{1}$ is with the property $P$, then $\operatorname{inc}(G) \leq \operatorname{inc}\left(G_{1}\right) \leq n-1$. Otherwise let $V_{1}\left(G_{1}\right)=\left\{v \in V\left(G_{1}\right) \mid d_{G_{1}}(v)=n-3\right\}$ and $V_{2}\left(G_{1}\right)=V\left(G_{1}\right) \backslash\left\{V_{1}\left(G_{1}\right) \cup\left\{v_{1}\right\}\right\}$. If $V_{2}\left(G_{1}\right)=\varnothing$, then inc $\left(G_{1}\right) \leq \Delta\left(G_{1}\right)+2$. If $V_{2}\left(G_{1}\right) \neq$ $\varnothing$, then $\left|V_{2}\left(G_{1}\right)\right| \geq 3$; there exists two vertices $u_{1}^{\prime}, v_{1}^{\prime}$ in $V_{2}\left(G_{1}\right)$ such that $u_{2}^{\prime} v_{1}^{\prime} \notin E\left(G_{1}\right)$. Let $G_{2}=G_{1}+u_{2}^{\prime} v_{2}^{\prime}$. If $G_{2}$ is not with the property $P$, then $\left|V_{2}\left(G_{2}\right)\right| \geq 3$ when $V_{2}\left(G_{2}\right) \neq \varnothing$. We can also construct graph $G_{3}$ that is not with the property $P$. In that way, we can obtain a serial of graphs $G, G_{1}, G_{2}, \ldots, G_{k}, \ldots$ such that all the graphs are not with the property $P$ and $\left|V_{2}\left(G_{k}\right)\right| \geq 3$. Let $D(G)=\sum_{v \in G} d(v)$, then $D(G) \leq D\left(G_{1}\right) \leq D\left(G_{2}\right) \leq \cdots \leq D\left(G_{k}\right) \leq$ … Because $G$ is the finite graph, there exists a graph $G_{k_{0}}$ such that $\left|V_{2}\left(G_{k_{0}}\right)\right|=3$. Suppose that $V_{2}\left(G_{k_{0}}\right)=\left\{u_{1}, u_{2}, u_{3}\right\}$ and $v^{\prime} \in V_{1}\left(G_{k_{0}}\right)$, then $V_{2}\left(G_{k_{0}}\right)=N\left(v^{\prime}\right)$. Thus $d_{G_{k_{0}}}\left(u_{1}\right)=$ $d_{G_{k_{0}}}\left(u_{2}\right)=d_{G_{k_{0}}}\left(u_{3}\right)=n-4$, and $u_{1}, u_{2}, u_{3}$ are without edge and adjacent to each other. Let $\hat{G}=G_{k_{0}}+u_{1} u_{2}$, then $u_{1}, u_{3} \in \hat{G} \backslash v_{1}, d\left(u_{1}\right)=n-3, d\left(u_{3}\right) \leq n-4$, and $u_{1} u_{3} \notin E(\hat{G})$, then $\hat{G}$ is with the property $P$, thus $\operatorname{inc}(G) \leq \operatorname{inc}\left(G_{1}\right) \leq \operatorname{inc}\left(G_{2}\right) \leq \cdots \leq \operatorname{inc}\left(G_{k_{0}}\right) \leq \operatorname{inc}(\hat{G}) \leq n-1$. The proof is complete.

Theorem 2.14. Let $u, v \in V(G)$ such that $u v \notin E(G)$ and $N_{G}(u)=N_{G}(v)$, then $\operatorname{inc}(G) \geq$ $\Delta+2$.

Proof. The proof is by contradiction. Suppose that the graph $G$ has an $(\Delta+1)$-incidence coloring with color set $C=\{1,2, \ldots, \Delta+1\}$. Let $N_{G}(u)=\left\{x_{1}, x_{2}, \ldots, x_{\Delta}\right\}$ and $N_{G}(v)=\left\{y_{1}\right.$, $\left.y_{2}, \ldots, y_{\Delta}\right\}$. Then each of the incidences $\left(x_{i}, x_{i} u\right)(1 \leq i \leq \Delta)$ is colored the same, as are the incidences $\left(y_{i}, y_{i} v\right)$. Without loss of generality, suppose $k$ the color that $\left(y_{i}, y_{i} v\right)$ has. Because $N_{G}(u)=N_{G}(v)$ and $\left(u, x_{1} x_{1}\right)$ is adjacent to $\left(y_{1}, y_{1} v\right)$, then $\left(u, u x_{1}\right)$ has a color other than $k$. Because $\left(u, u x_{2}\right)$ is adjacent to $\left(y_{2}, y_{2} v\right), \ldots,\left(u, u x_{\Delta}\right)$ which is adjacent to $\left(y_{\Delta}, y_{\Delta} v\right)$, then $\left(u, u x_{2}\right), \ldots,\left(u, u x_{\Delta}\right)$ also has a color other than $k$, respectively. Further, the $\Delta$ incidences $\left(u, u x_{i}\right)(1 \leq i \leq \Delta)$ have different colors, so the color $k$ is different from that of incidences $\left(u, u x_{i}\right)$. On the other hand, $\left(y_{1}, y_{1} v\right)$ and $\left(x_{1}, x_{1} u\right)$ are neighborly incidences, so the color $k$ is different from that of $\left(x_{1}, x_{1} u\right)$. Thus $k \notin C$, this gives a contradiction! Hence inc $(G) \geq \Delta+2$. 


\section{The incidence chromatic number of complete $k$-partite graph}

Theorem 3.1. Let $G=K_{n_{1}, n_{2}, \ldots, n_{k}}$ be a complete $k$-partite graph $(k \geq 2)$. Then

$$
\operatorname{inc}(G)= \begin{cases}\Delta+1, & \Delta(G)=n-1, \\ \Delta(G)+2, & \text { otherwise. }\end{cases}
$$

Proof. Let $V(G)=V_{1} \cup V_{2} \cup \cdots \cup V_{k}$ and $\left|V_{i}\right|=n_{i}(i=1,2, \ldots, k) . V_{i}$ is the $i$-part vertex set and $V_{i}=\left\{v_{1}^{i}, v_{2}^{i}, \ldots, v_{n_{i}}^{i}\right\}(i=1,2, \ldots, k)$. Without loss of generality, we let $n_{1} \geq$ $n_{2} \geq \cdots \geq n_{k}$. Thus $\Delta(G)=\sum_{m=1}^{k-1} n_{m}$. The proof can be divided into the following two cases.

Case 3. There exists $i \in\{1,2, \ldots, k\}$ such that $n_{i}=1$. We let the vertex set of $G$ be $V(G)=$ $\left\{v_{i}, v_{2}, \ldots, v_{m}\right\}$, where $m=\sum_{i=1}^{k} n_{i}$. By Lemma 2.6, it easy to draw the conclusion.

Case 4. $n_{i} \geq 2(1 \leq i \leq k)$. To complete the proof, we give an incidence coloring just with $\Delta+2$ colors firstly.

For $j, t=1,2, \ldots, k, i=1,2, \ldots, n_{j}$, and $s=1,2, \ldots, n_{t}$, we let

$$
\sigma\left(v_{i}^{j}, v_{i}^{j} v_{s}^{t}\right)= \begin{cases}\sum_{m=0}^{t-1}\left(n_{m}+s\right), & i \neq s, t<j \text { or } i=s, t>j, \\ \sum_{m=0}^{t-2}\left(n_{m}+s\right), & i \neq s, t>j \text { or } i=s, t<j, \\ \Delta+1, & i=s, t=1, \\ \Delta+2, & i=s, t=k .\end{cases}
$$

To complete the proof, it suffices to prove that $G$ cannot be colored with $\Delta+1$ colors. It is obvious that each of the vertices in $V_{1}$ is the maximum-degree vertex. For $n_{1} \geq$ 2 , let $u, v \in V_{1}$, then $u v \notin E(G)$ and $N(u) \neq N(v)$. Hence $\operatorname{inc}(G) \geq \Delta+2$ follows from Theorem 2.14. Therefore $\operatorname{inc}(G)=\Delta+2$, and the proof is completed.

By Theorem 3.1, it is easy to obtain the theorem in $[3,4]$. In fact, the incidence coloring $\sigma$ given to determine the incidence chromatic number for complete 3-partite graphs is a special case of the coloring above. Hence, we obtain some corollaries as follows.

Corollary 3.2. Let $K_{n}$ be complete graph. Then $\operatorname{inc}\left(K_{n}\right)=n$.

The incidence coloring of $K_{3,4}$ and $K_{5}$ is given in Figure 3.1.

\section{Incidence chromatic number of three families of graphs}

The planar graph $Q_{n}$, which is called triangular prism, is defined by $Q_{n}=G(V(G), E(G))$, where the vertex set $V(G)=u_{1}, u_{2}, \ldots, u_{n} \cup v_{1}, v_{2}, \ldots, v_{n}$, and the edges set $E\left(Q_{n}\right)$ consists 


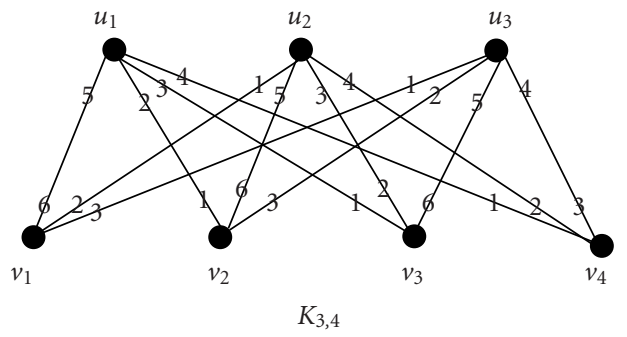

(a)

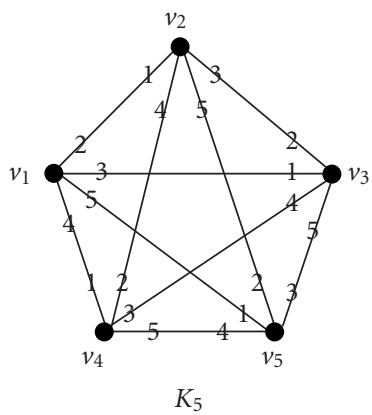

(b)

Figure 3.1. An incidence coloring of $K_{3,4}$ and $K_{5}$, respectively.

of two $n$-cycles $u_{1}, u_{2}, \ldots, u_{n}$ and $v_{1}, v_{2}, \ldots, v_{n}$, and $2 n$ edges $\left(u_{i}, v_{i}\right),\left(u_{i}, v_{i+1}\right)$ for all $i \in$ $[n]\left(v_{1}=v_{n+1}\right)$.

Theorem 4.1. For any integer $n \geq 3$,

$$
\operatorname{inc}\left(Q_{n}\right)= \begin{cases}\Delta+1=5, & n=0 \bmod (5), \\ \Delta+2=6, & \text { otherwise. }\end{cases}
$$

Proof. Because $\Delta(G)=4$, we know that $\operatorname{inc}\left(Q_{n}\right) \geq \Delta+1=5$. When $n=5 k, k \geq 1$, we give a 5 -incidence coloring $\sigma$ of $Q_{5 k}$. For $i=1,2, \ldots, 5 k$, let $\left(u_{i}, u_{i} u_{i}^{*}\right)$ be the incidence set $\left\{u_{i}, u_{i} w \mid w=v_{i+1}, u_{i \pm 1}, v_{i}\right\}$. Let

$$
\begin{gathered}
\sigma\left(u_{i}, u_{i} u_{i}^{*}\right)=\{1+2(i-1)(\bmod 5), 2+2(i-1)(\bmod 5), \\
3+2(i-1)(\bmod 5), 4+2(i-1)(\bmod 5)\}, \\
\sigma\left(v_{i \pm 1}, v_{i \pm 1} v_{i}\right)=\sigma\left(u_{i}, u_{i} v_{i}\right), \quad \sigma\left(w, w u_{i}\right)=\sigma\left(u_{i m p 1}, u_{i \mp 1} u_{i}\right), \\
\sigma\left(v_{i}, v_{i} u_{i}\right)=\sigma\left(u_{i+1} u_{i+1} v_{i+1}\right) .
\end{gathered}
$$

It is easy to see that the coloring above is a proper 5-incidence coloring of $Q_{n}$. Thus, we can only consider the case $n \neq 5 k$. We will first prove that $Q_{n}$ is 6-incidence colorable by explicitly giving a 6-incidence coloring $\sigma$ of $Q_{n}$ for any integer $n \geq 3$. At last, we will give the proof that $Q_{n}$ cannot be incidence coloring just with colors $1,2,3,4,5$. The proof can be divided into the following three cases.

Case 5. $n=3 k(k \geq 1)$. Let $i=3 s+t(t \leq 2), i=1,2, \ldots, n$, then $Q_{n}$ has an incidence coloring using 6 colors from the color set $C=\{1,2, \ldots, n+r+1\}$, as follows: for $i=1,2, \ldots, n$, 
let

$$
\begin{aligned}
& \sigma\left(v_{i}, v_{i} v_{i+1}\right)=\sigma\left(u_{i}, u_{i} u_{i+1}\right)= \begin{cases}t, & t \neq 0, \\
3, & t=0,\end{cases} \\
& \sigma\left(v_{i}, v_{i} v_{i-1}\right)=\sigma\left(u_{i}, u_{i} u_{i-1}\right)=t+1, \\
& \sigma\left(u_{i}, u_{i} v_{i+1}\right)=\sigma\left(v_{i}, v_{i} u_{i-1}\right)=\sigma\left(u_{i}, u_{i} u_{i+1}\right)+3, \\
& \sigma\left(u_{i}, u_{i} v_{i}\right)=\sigma\left(v_{i+1}, v_{i+1} u_{i+1}\right)=\sigma\left(u_{i+1}, u_{i+1} u_{i}\right)+3 .
\end{aligned}
$$

Case 6. $n=3 k+1(k \geq 1)$. Let $i=3 s+t(t \leq 2)$. For $i=1,2, \ldots, n$, let

$$
\begin{gathered}
\sigma\left(v_{i}, v_{i} v_{i+1}\right)=\left\{\begin{array}{ll}
3, & t=0, \\
4, & i=1, \\
5, & i=2, \\
t, & \text { otherwise }
\end{array} \quad \sigma\left(u_{i}, u_{i} u_{i+1}\right)= \begin{cases}3, & t=0, \\
6, & i=1, \\
t, & \text { otherwise },\end{cases} \right. \\
\sigma\left(v_{i}, v_{i} v_{i-1}\right)=\left\{\begin{array}{ll}
6, & i=1, \\
2, & i=2, \\
t+1, & \text { otherwise }
\end{array} \quad \sigma\left(u_{i}, u_{i} u_{i-1}\right)= \begin{cases}5, & i=1, \\
4, & i=n, \\
t+1, & \text { otherwise, }\end{cases} \right. \\
\sigma\left(u_{i}, u_{i} v_{i}\right)=\sigma\left(v_{i+1}, v_{i+1} u_{i+1}\right)= \begin{cases}\sigma\left(u_{i+1}, u_{i+1} u_{i}\right)+3, & i \neq 1, n, \\
5, & i=n,\end{cases} \\
\sigma\left(u_{1} u_{1} v_{1}\right)=\sigma\left(v_{2} v_{2} v_{1}\right)=2, \quad \sigma\left(v_{1} v_{1} u_{3}\right)=3 .
\end{gathered}
$$

Case 7. $n=3 k+2(k \geq 1)$. Let $i=3 s+t(t \leq 2)$, for $i=1,2, \ldots, n$, and $w_{n+1}=w_{1}, w=u, v$; $w_{0}=w_{n}, w=u, v$. We let

$$
\begin{aligned}
& \sigma\left(u_{i}, u_{i} u_{i+1}\right)= \begin{cases}3, & t=0, \\
5, & i=n, \\
6, & i=1, \\
t, & \text { otherwise, }\end{cases} \\
& \sigma\left(u_{i}, u_{i} v_{i}\right)=\sigma\left(v_{i+1}, v_{i+1} u_{i+1}\right)= \begin{cases}\sigma\left(u_{i+1}, u_{i+1} u_{i}\right)+3, & i \neq 1, n, \\
3, & i=1, \\
5, & i=n,\end{cases}
\end{aligned}
$$




$$
\begin{aligned}
& \sigma\left(v_{i}, v_{i} v_{i+1}\right)= \begin{cases}2, & i=n, \\
3, & t=0, \\
4, & i=1, \\
t, & \text { otherwise }\end{cases} \\
& \sigma\left(u_{i}, u_{i} v_{i}\right)= \sigma\left(u_{i}, u_{i} u_{i+1}\right)+3= \begin{cases}2, \\
5, \\
\sigma\left(u_{i}, u_{i} u_{i+1}\right)+3, & i \neq 1, n,\end{cases} \\
& \sigma\left(u_{i}, u_{i} u_{i-1}\right)= \sigma\left(v_{i}, v_{i} v_{i-1}\right)= \begin{cases}1, & i=1, \\
t+1, & \text { otherwise },\end{cases} \\
& \sigma\left(v_{i}, v_{i} u_{i-1}\right)= \begin{cases}\sigma\left(u_{i}, u_{i} u_{i+1}\right)+3, & i \neq 1, n \\
2, & i=n, \\
6, & i=1,\end{cases} \\
& \sigma\left(u_{i}, u_{i} v_{i+1}\right)= \begin{cases}\sigma\left(u_{i}, u_{i} u_{i+1}\right)+3, & i \neq 1, n, \\
2, & i=n, \\
4, & i=1,\end{cases} \\
& \sigma\left(v_{i}, v_{i} u_{i-1}\right)= \begin{cases}\sigma\left(u_{i}, u_{i} u_{i+1}\right)+3, & i \neq 1, n, \\
2, & i=n, \\
6, & i=1 .\end{cases}
\end{aligned}
$$

It is easy to show that $Q_{n}$ is 6-incidence colorable. To complete the proof, it remains to be shown that there do not exist an incidence coloring using only 5 colors. Assume, on the contrary, that $Q_{n}$ is 5-incident colorable. For each vertex $v_{i} \in Q_{n}, d\left(v_{i}\right)=\Delta\left(Q_{n}\right)$. Thus, four incidences $\left(u_{i}, u_{i} v_{i}\right),\left(u_{i-1}, u_{i-1} v_{i}\right),\left(v_{i \pm 1}, v_{i \pm 1} v_{i}\right)$ have the same color, without loss of generality, 1 . For $i=1,2, \ldots, n$, the case is the same. Because there are 5 colors that can be used in incidence coloring, and the degree of each vertex $v_{i}$ in cycle $v_{1} v_{2} \cdots v_{n} v_{1}$ is 4 , thus the two incidences $\left(v_{i}, v_{i} v_{i+1}\right)$ and $\left(v_{i+4}, v_{i+4} v_{i+5}\right)$ (or $\left.\left(v_{i-4}, v_{i-4} v_{i-5}\right)\right)$ have the same color. If $n \neq 5 k$, form the proof above, it is easy to obtain a contradiction. Thus, we have completed the prove.

Theorem 4.2. Let $G$ be a Hamilton graph with order $n \geq 3$ and degree $\Delta \leq 3$. Then $\operatorname{inc}(G) \leq \Delta+2$.

Proof. When $\Delta \leq 2$, by Lemma $2.2, \operatorname{inc}(G) \leq \Delta+2$. When $\Delta=3$, by Lemma 2.3 , we can only consider the case $d(v)=3(\forall v \in V(G))$. Let $\left\{v_{1}, v_{2}, \ldots, v_{n}, v_{1}\right\}$ be the Hamilton cycle and $S=E(G) \backslash\left\{v_{i} v_{i+1} \mid 1 \leq i \leq n-1\right\}$. The proof can be divided into the following three cases.

Case 8. $n=0 \bmod (3)$. For $i=1,2, \ldots, n$, we let $\sigma\left(v_{i}, v_{i} v_{i+1}\right)=2 i-1(\bmod 3)$ and $\sigma\left(v_{i+1}\right.$, $\left.v_{i+1} v_{i}\right)=2 i(\bmod 3)$, where $v_{n+1}=v_{1}$. Because the edges $e \in S$ form a matching, thus we can incidence color the incidence uncolored with two new colors 3,4 . Then, we have given $G$ an incidence coloring with colors $0,1, \ldots, 4$. 
812 The incidence chromatic number of some graph

Case 9. $n \neq 0 \bmod (3)$. Let $v_{j} \in A_{v_{1}}(j \neq 1, n)$ and $v_{k} \in A_{v_{n}}(n \neq 1, n-1)$. For $i=1,2, \ldots, n$ and $v_{n+1}=v_{1}$, we let

$$
\begin{aligned}
& \sigma\left(v_{i}, v_{i} v_{i+1}\right)= \begin{cases}2 i-1(\bmod 3), & i \neq 1, j, \\
4, & i=j=k+1, \\
3, & \text { otherwise, }\end{cases} \\
& \sigma\left(v_{i+1}, v_{i+1} v_{i}\right)= \begin{cases}2 i(\bmod 3), & i \neq 1, j-1, \\
3, & i=j-1=k, \\
4, & \text { otherwise, }\end{cases} \\
& \sigma\left(v_{j}, v_{j} v_{1}\right)= \begin{cases}1, & n=1 \bmod (3) \text { and } j=1 \bmod (3), \\
0, & n=2 \bmod (3) \text { and } j \neq 0 \bmod (3), \\
2, & \text { otherwise, }\end{cases} \\
& \sigma\left(v_{1}, v_{1} v_{j}\right)=n-1 \bmod (3) .
\end{aligned}
$$

Since the edges $e \in S \backslash\left\{v_{1} v_{k}\right\}$ form a matching, thus we can incidence color the incidence uncolored with two new colors 3,4 . Thus, we have given $G$ an incidence coloring with colors $0,1, \ldots, 4$.

The plane check graph $C_{m, n}$ is defined by $V\left(C_{m, n}\right)=\left\{v_{i, j} \mid i \in[m] ; j \in[n]\right\} ; E\left(C_{m, n}\right)=$ $\left\{v_{i, j} v_{i, j+1} \mid i \in[m] ; j \in[n-1]\right\} \cup\left\{v_{i, j} v_{i+1, j} \mid i \in[m-1] ; j \in[n]\right\}$, which is the Cartesian product of path $P_{m}$ and $P_{n}$,

Theorem 4.3. For plane graph $C_{m, n}$, we have inc $\left(C_{m, n}\right)=5$.

Proof. $\Delta\left(C_{m, n}\right)=4$, then $\operatorname{inc}\left(C_{m, n}\right) \geq 5$. We now give a 5-incidence coloring $\sigma$ of $C_{m, n}$ as follows: $(i \in[m] ; j \in[n])$

$$
\begin{aligned}
\sigma\left(v_{i, j}, v_{i, j} v_{i, j+1}\right) & =j+3(i-1)(\bmod (5))(j \neq n), \\
\sigma\left(v_{i, j+1}, v_{i, j+1} v_{i, j}\right) & =j+4(i-1)(\bmod (5))(j \neq n), \\
\sigma\left(v_{i, j}, v_{i, j} v_{i+1, j}\right) & =j+2(i-1)(\bmod (5))(i \neq m), \\
\sigma\left(v_{i+1, j}, v_{i+1, j} v_{i, j}\right) & =j+4(i-1)(\bmod (5))(i \neq m) .
\end{aligned}
$$

It is easy to see that the coloring above is an incidence coloring of $C_{m, n}$. Thus $\operatorname{inc}\left(C_{m, n}\right)=5$.

Remark 4.4. It is difficult to obtain the incidence chromatic number for some graphs. We have presented a hybrid genetic algorithm for the incidence coloring on graphs in [6]. The experimental results indicate that a hybrid genetic algorithm can obtain solutions of excellent quality of problem instances with different size.

\section{Acknowledgment}

This work is supported by China National Science Foundation. 


\section{References}

[1] I. Algor and N. Alon, The star arboricity of graphs, Discrete Math. 75 (1989), no. 1-3, 11-22.

[2] J. A. Bondy and U. S. R. Murty, Graph Theory with Applications, American Elsevier Publishing, New York, 1976.

[3] R. A. Brualdi and J. J. Q. Massey, Incidence and strong edge colorings of graphs, Discrete Math. 122 (1993), no. 1-3, 51-58.

[4] D. L. Chen, X. K. Liu, and S. D. Wang, The incidence chromatic number and the incidence coloring conjecture of graph, Mathematics in Economics 15 (1998), no. 3, 47-51.

[5] B. Guiduli, On incidence coloring and star arboricity of graphs, Discrete Math. 163 (1997), no. 13, 275-278.

[6] X. K. Liu and Y. Li, Algorithm for graph incidence coloring base on hybrid genetic algorithm, Chinese J. Engrg. Math. 21 (2004), no. 1, 41-47.

Liu Xikui: College of Information \& Engineering, Shandong University of Science and Technology, Qingdao 266510, Shandong, China

Current address: School of Professional Technology, Xuzhou Normal University, Xuzhou 221011, Jiangsu, China

E-mail address: xkliubs@eyou.com

Li Yan: College of Information \& Engineering, Shandong University of Science and Technology, Qingdao 266510, Shandong, China

E-mail address: liyan7511@eyou.com 


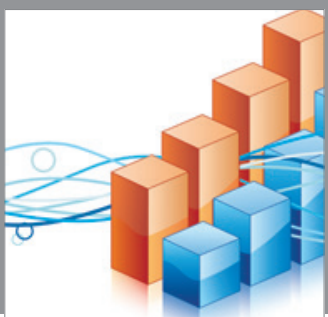

Advances in

Operations Research

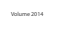

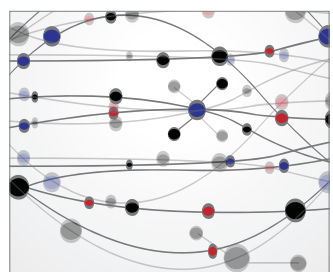

\section{The Scientific} World Journal
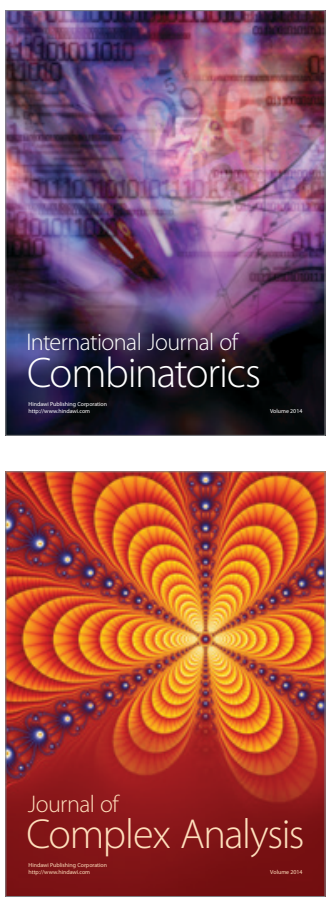

International Journal of

Mathematics and

Mathematical

Sciences
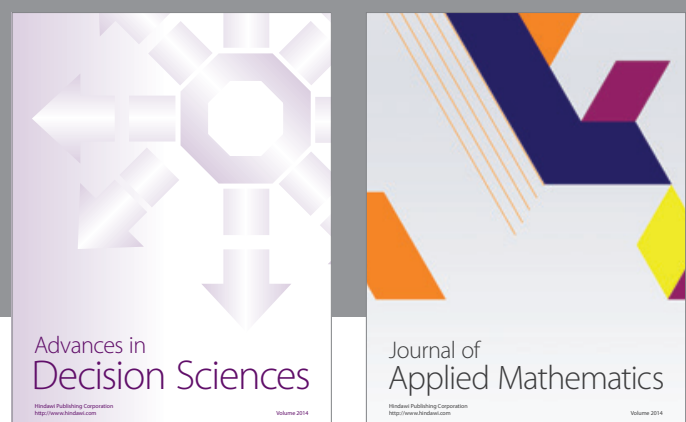

Journal of

Applied Mathematics
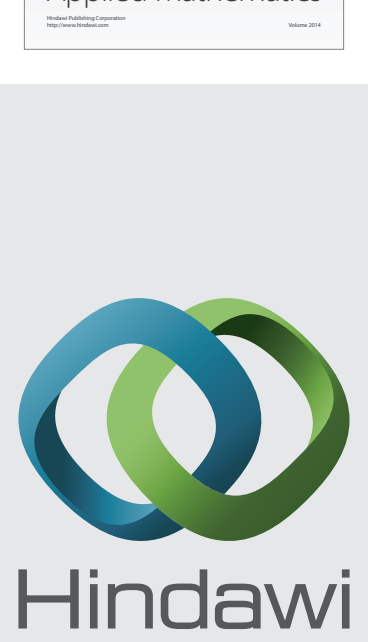

Submit your manuscripts at http://www.hindawi.com
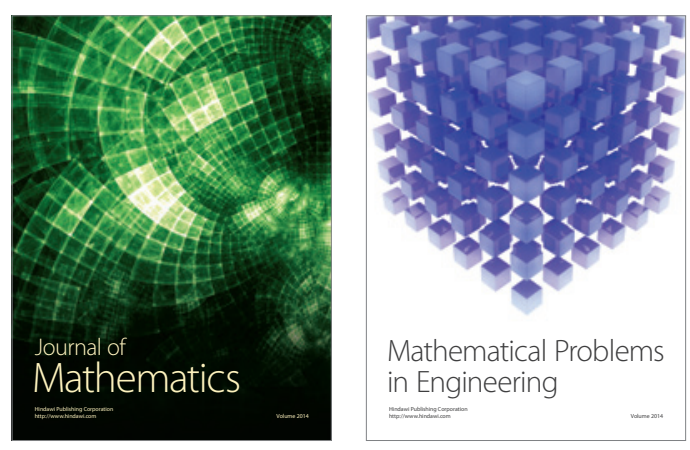

Mathematical Problems in Engineering
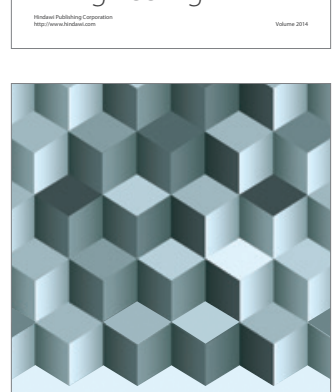

Journal of

Function Spaces
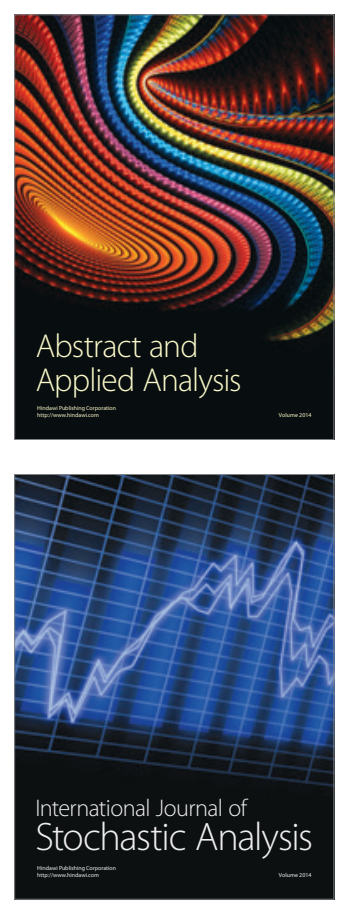

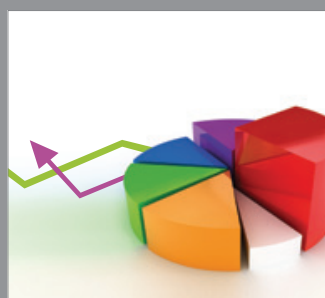

ournal of

Probability and Statistics

Promensencen
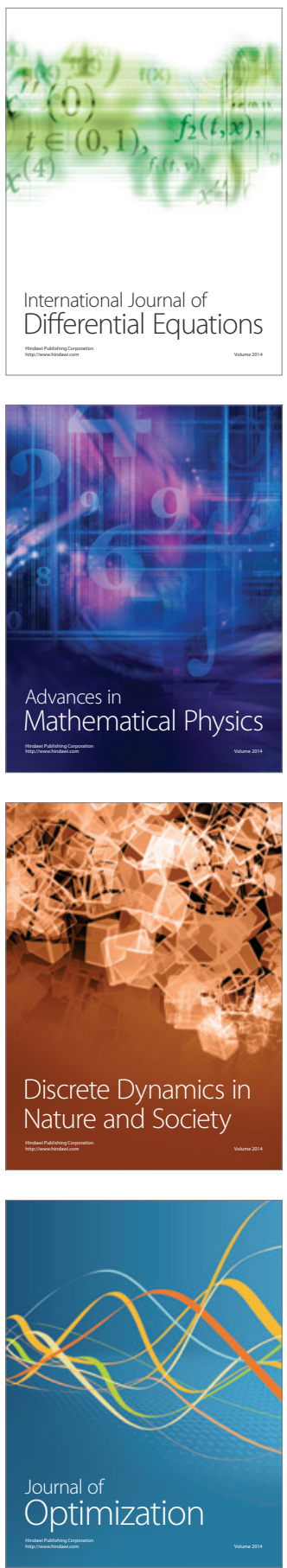